\title{
Comparison of Core Muscle Activation Between a Prone Bridge and 6-RM Back Squats
}

\author{
by \\ Roland van den Tillaar ${ }^{1}$, Atle Hole Saeterbakken ${ }^{2}$
}

The purpose of this study was to compare core muscle activation during a prone bridge (plank) until failure and 6-RM back squats. Twelve resistance-trained males (age $23.5 \pm 2.6$ years, body mass $87.8 \pm 21.3 \mathrm{~kg}$, body height $1.81 \pm 0.08 \mathrm{~m})$ participated in this study. Total exercise time and EMG activity of the rectus abdominis, external abdominal oblique and erector spinae were measured during 6-RM back squats and a prone bridge with a weight of $20 \%$ of participants' body mass on their lower back. The main findings showed non-significant differences between the exercises in the rectus abdominis or external oblique, but greater erector spinae activation in squatting. Furthermore, in contrast to the prone bridge, the erector spinae and rectus abdominis demonstrated increasing muscle activation throughout the repetitions while squatting, whereas the prone bride demonstrated increasing external oblique activation between the beginning and the middle of the set. It was concluded that since squatting resulted in greater erector spine activation, but similar rectus abdominis and oblique external activation as the prone bridge, high-intensity squats rather than isometric low intensity core exercises for athletes would be recommended.

Key words: EMG, core stability, core strength, performance.

\section{Introduction}

Adequately developed core strength is widely regarded as an important prerequisite for sports performance (Behm et al., 2015; Borghuis et al., 2008; Kibler et al., 2006). There is evidence that better core strength can lead to reduced low back pain and improve sports performance (McGill, 2001; Saeterbakken et al., 2011). Well-developed core strength may contribute to stabilizing the core by stiffening the torso (Roth et al., 2016) and transferring the energy of the legs to the upper extremities (Kibler et al., 2006).

There are countless exercises designed to target core muscles (Behm et al., 2015; Borghuis et al., 2008; Kibler et al., 2006). In the last decade, several different approaches and variations of core exercises have been developed to increase core muscle activation. Greater muscle activation is associated with recruitment of type II muscle fibers, which have greater potential than type I muscle fibers to improve strength and hypertrophy (ACSM, 2009). Muscle atrophy or weak core muscles may be associated with increased risk of low back pain. However, the approaches can be divided into two conditions: training core muscles isometrically and in isolation (Monfort-Panego et al., 2009) or training core muscles dynamically and in an integrated manner (Kibler et al., 2006).

The prone bridge (plank) is one of the most frequently used exercises to isolate the abdominal muscles. The exercise is performed in different variations (i.e. with a longer lever and/or a posterior tilt (Schoenfeld et al., 2014) or on unstable surfaces (Czaprowski et al., 2014; Snarr and Esco, 2014)).

Several studies have compared core muscle activation during different isometric core exercises (Clark et al., 2003; Oliver et al., 2010). In

1 - Department of Sports Science and Physical Education, Nord University, Levanger, Norway.

2 - Department of Teacher Education and Sports, Western Norwy University of Applied Sciences, Sogndal, Norway 
most of these studies, core muscle activity was normalized against maximal voluntary contractions (MVC) of each muscle (Czaprowski et al., 2014; Escamilla et al., 2006; Roth et al., 2016; Snarr and Esco, 2014; Sumiaki et al., 2013), with varying and contradictory EMG results (Schoenfeld et al., 2014).

However, isometric, single-muscle, and isolated core exercises from a prone position may not transfer effectively to the predominately erect dynamic activities of daily living and sports performance (Borghuis et al., 2008; Lederman, 2010). No previous studies provided a clear demonstration that an isolated and isometric contraction (i.e. prone bridge) is superior to dynamic core exercises. For example Escamilla et al. (2006) and Gottschall et al. (2013) demonstrated greater core muscle activation while performing dynamic movements compared to isolated and isometric contractions during core exercises. Furthermore, dynamic multi-joint resistancetraining exercises (i.e. squats and dead lifts) may integrate and mimic core muscle activation that is more similar to the activation patterns in sports and daily activities.

Lately, some studies have compared isolated core exercises with integrated multi-joint free-weight resistance exercises (i.e. squat) and demonstrated lower, greater or similar muscle activation observed when analyzing these two types of exercise (Comfort et al., 2011; Ekstrom et al., 2007; Hamlyn et al., 2007; Nuzzo et al., 2008; Snarr and Esco, 2013). Yet, all these studies have several limitations. Firstly, all the abovementioned studies used only physically active subjects who had no previous experience with weight training and core exercises. Thus, it is not certain whether these findings would also occur in resistance-trained subjects. Secondly, Nuzzo et al. (2008) examined core exercises on an unstable surface (Swiss ball) and not a stable surface with free-weight exercises. It is well documented that an unstable surface increases muscle activation compared to a stable one (Behm and Colado, 2012). It is therefore uncertain whether the stability requirement may have resulted in greater core muscle activation than a traditional approach in the prone bridge exercise. Thirdly, Snarr and Esco (2013) and Ekstrom et al. (2007) used body weight or low external resistance in the integrated exercises, which is not comparable with training approaches used among athletes. Finally, the isometric low-load core strength exercise was only conducted for $30 \mathrm{~s}$, not until exhaustion, which could influence the results (Tong et al., 2014). Previous studies that investigated neuromuscular fatigue due to exhaustion during strength training showed conflicting results varying from increased muscle activation (Brennecke et al., 2009; Gentil et al., 2007), no difference (Lindström et al., 2006), to decreased muscle activation during a set (Gerdle et al., 2000). However, to recruit type II muscle fibers which have the greatest potential to improve strength and muscle hypertrophy, the movement has to be performed very rapidly or to exhaustion (ACSM, 2009). Therefore, the effects of performing the prone bridge or squats to exhaustion are not well documented.

To the best of our knowledge, no previous study has compared core muscle activation while performing a dynamic high-intensity resistance exercise (squat) integrating core muscles with an isolated and isometric core exercise (prone bridge) performed to exhaustion. Therefore, the primary aim of this study was to compare core muscle activity between the prone bridge exercise and back squats in experienced resistance-trained subjects; secondly, to investigate muscle activity when performing these exercises to exhaustion. Based upon the findings of Hamlyn et al. (2007), it was hypothesized that the EMG activity of the erector spinae would be higher during the squat exercise compared to the prone bridge and that the abdominal muscles would present higher activation in the prone bridge than the squats since this exercise was specifically designed to target abdominal muscles while the squats were more oriented towards strengthening lower limbs and the erector spinae. Furthermore, it was hypothesized that muscle activity would increase during both exercises due to fatigue (Tong et al., 2014).

\section{Methods}

To compare the effects of 6-RM back squats and the prone bridge on core muscle activity, a within-subjects design was used in which each subject performed both exercises. 6$\mathrm{RM}$ was used because it is a typical training load to increase maximal strength (ACSM, 2009). For the prone bridge, a weight of approximately $20 \%$ of body mass was added on the lower back to 
increase core muscle activity and to avoid limitation of performance due to fatigue of the extremities (Roth et al., 2016). These two exercises were chosen since both are very popular exercises in strength and core training. The dependent variables were the total lifting time and the EMG activity of the rectus abdominis, external oblique, and erector spinae during the two exercises.

\section{Participants}

Twelve resistance-trained males (age 23.5 \pm 2.6 years, body mass $87.8 \pm 21.3 \mathrm{~kg}$, body height $1.81 \pm 0.08 \mathrm{~m})$ participated in this study. Each subject had at least two-year experience of resistance training in the prone bridge and weight trained at least twice a week. Seven of the subjects were competitive power-lifters, whereas the others were sprinters at the national level. Inclusion criteria were no recent injuries or pain, which could reduce maximal performance, and the ability to lift 1.2 times one's own body weight in a full squat with a good full squatting technique, which was evaluated by an experienced weight-lifting coach. These factors were all essential to ensure the subjects had a proper upper body posture and muscle activation during the lifts. The subjects did not perform any resistance exercises targeting lower extremities for 48 hours before the test. All subjects signed a written informed consent form outlining the risk factors and their right to withdraw from the research at any time without stating a reason. The study was approved by the local committee for medical research ethics and complied with the current ethical standards in sports and exercise research.

\section{Test procedures}

Before the test, posture and body mass were evaluated, which was necessary to calculate the weight that would be placed on the lower back of the subjects during the prone bridge ( $20 \%$ of body mass). The subjects were experienced resistance-trained athletes who were familiar with both testing exercises. Therefore, no familiarization test, one repetition maximum (1$\mathrm{RM})$ test, or 6-RM test was performed, and only subjects' self-reported 1-RM and 6-RM weights were used. Before the test started, the subjects warmed up with 20 repetitions (reps) at $25 \%$ of their self-reported 1-RM, followed by 10 reps at $50 \%$ and 8 reps at $70 \%$ of the self-reported a-RM (van den Tillaar et al., 2014). Then, two sets of six lifts at 6-RM were performed to establish if core muscle activity behaved the same and to calculate the interclass coefficient $\operatorname{ICC}(3,1))$. After the two sets of squatting, one trial of the prone bridge was performed with weights of approximately $20 \%$ of body mass on the lower back until failure (Figure 1). This extra weight on the lower back was used to prevent the subjects from having to stop the exercise due to fatigue in other muscle groups like in the shoulders. Between each sets and exercise, the subjects were given four to five minutes of rest to provide for optimal performance and avoid fatigue (Rahimi, 2005).

The subjects performed the squat with their feet placed in their preferred position (to avoid extra stress upon the subject and to increase the external validity towards training), and the position of the feet was measured to maintain the identical foot position during the exercise. From this position, with a barbell on the upper part of the shoulder, the subject flexed the knees down to a depth where the hip crease dropped below the patella for all repetitions. This position was found using a protractor. A horizontal rubber band was used to identify this lower position during the tests, which the subjects had to touch with the proximal part of their hamstring before starting the ascending movement (Saeterbakken et al., 2016; van den Tillaar et al., 2014). The ascending movement was performed with the highest intensity under full control in every repetition.

\section{Instrumentation}

Musclelab (MuscleLab 6000 system, Ergotest AS Porsgrund, Norway) was used to measure EMG activity in the erector spinae, rectus abdominis, and abdominal external oblique according to the recommendations of SENIAM (Hermens et al., 2000) and as used in other studies (van den Tillaar, 2015; van den Tillaar et al., 2014). Before positioning the electrodes over each muscle, the skin was shaved, abraded, and cleaned with isopropyl alcohol to reduce skin impendence. To strengthen the signal, conductive gel (Signa Gel, Parker Laboratories Inc, USA) was applied to self-adhesive electrodes (Dri-Stick Silver circular sEMG Electrodes AE-131, NeuroDyne Medical, USA). The electrodes were placed on the subject's dominant side. The raw EMG signals, sampled at $1000 \mathrm{~Hz}$, were amplified and filtered using a preamplifier located as close to the pickup point as possible. The signals were 
high-pass and low-pass (600, $8 \mathrm{~Hz}$ ) filtered, rectified, integrated, and converted to root mean square (RMS) signals using a hardware circuit network (frequency response $450 \mathrm{kHz}$, averaging constant $12 \mathrm{~ms}$, total error $\pm 0.5 \%$ ) with a common rejection rate of $106 \mathrm{~dB}$ (van den Tillaar and Saeterbakken, 2014).

During the 6-RM squats, a linear encoder (ET-Enc-02, Ergotest Technology AS, Langesund, Norway) connected to the barbell measured the vertical position and velocity at $0.075-\mathrm{mm}$ resolution and counted the pulses at $10-\mathrm{ms}$ intervals (Arnason et al., 2004). The velocity of the barbell was calculated using a five-point differential filter with software Musclelab V10.4 (Ergotest technology AS). Further, the linear encoder was used to identify the beginning and the end of each of the six repetitions. For the prone bridge, the linear encoder was manually triggered to identify the beginning and the end of exercise. Finally, the linear encoder was synchronized with the EMG recordings and made it possible to calculate the specific EMG activity for each repetition of the squat or for the prone bride exercise. The EMG data, velocity and vertical position were analyzed by the Musclelab 6000 using the software version V10.4 (Ergotest Technology AS).

Since both exercises were until complete exhaustion, it was chosen to compare the EMG activity in two ways. 1) Average RMS EMG activity of each muscle during the total lifting times of each exercise was calculated. The total lifting time during the squat was quantified from the first descending movement of the barbell until it was lifted up again to the starting position in the $6^{\text {th }}$ repetition. The total lifting time in the prone bridge was evaluated from the moment when the subjects with the added $20 \%$ of body mass on the lower back assumed the prone-bridge position until they could not hold this position anymore. 2) The average RMS EMG activity of each core muscle was measured during the first 10 $\mathrm{s}$, middle $10 \mathrm{~s}$, and the last $10 \mathrm{~s}$ during the prone bridge as well as during repetition 1, 4, and 6 during the squats, as quantified by the linear encoder, to investigate the development of the EMG activity of the three muscles in both exercises (Hamlyn et al., 2007). These periods and repetitions were chosen since they indicated approximately the start, the middle and the end of each exercise and thereby similar fatigue levels during each exercise.

\section{Statistical analyses}

To compare neuromuscular activity between the two exercises, a repeated analysis of variance (one-way ANOVA) design was used on each of the three muscles. In addition, to investigate the development of muscle activity during both exercises, a two-way ANOVA [(exercise: squat, planking) $x$ (time: start middle, end of exercise)] was used. Post-hoc comparisons with HolmBonferroni corrections were conducted to locate differences.

All results were presented as mean $\pm \mathrm{SD}$. The level of significance was set at $p<0.05$. Effect size was evaluated with $\eta^{2}$ (Eta partial squared), where $0.01<\eta^{2}<0.06$ constituted a small effect, a medium effect when $0.06<\eta^{2}<0.14$, and a large effect when $\eta^{2}>0.14$ (Cohen, 1988). Statistical analyses were performed using the SPSS version 22.0 (SPSS, Inc., Chicago, IL, USA).

\section{Results}

The lifted 6-RM load in the squat was 103 $\pm 31 \mathrm{~kg}$. No significant differences in the EMG activity for none of the measured muscles between the two squats were found ( $\mathrm{F} \leq 1.5, p \geq$ $0.239, \eta^{2} \geq 0.12$ ), with ICC EMG values of 0.94 or higher. Therefore, the average EMG activity of the two squats was compared with the EMG activity of the prone bridge. A significantly higher activity only in the erector spinae during the squats was found throughout the whole period compared with the prone bridge $\left(\mathrm{F}=18.4, p<0.001, \eta^{2}=\right.$ 0.82 ). For the other two muscles, no significant differences were found ( $\mathrm{F} \leq 1.5, p \geq 0.256, \eta^{2} \geq 0.10$, Figure 2).

The total lifting time was $27.7 \pm 5.6 \mathrm{~s}$, while the lifting time during the prone bridge $(92.8 \pm 58.2 \mathrm{~s})$ was significantly longer $(p=0.002)$. When the time-course during the two exercises was investigated, an effect of time was found for the external oblique $\left(\mathrm{F}=10.0, p=0.001, \eta^{2}=0.48\right)$ and rectus abdominis $\left(F=9.1, p=0.002, \eta^{2}=0.48\right)$, while no effect of time was found for the erector spinae $\left(F=1.5, p=0.24, \eta^{2}=0.12\right.$, Figure 3$)$. However, a significant interaction effect was found for the erector spinae $\left(\mathrm{F}=9.6, p=0.001, \eta^{2}=\right.$ $0.46)$, and a trend was found for the external oblique $\left(\mathrm{F}=2.7, p=0.087, \eta^{2}=0.20\right)$. Post-hoc comparison revealed that muscle activity 
significantly increased only for the external oblique from the first $10 \mathrm{~s}$ to the middle $10 \mathrm{~s}$ in the prone bridge, while activity increased in the squats from repetitions 1 to 4 for the rectus abdominis. For the erector spinae, EMG activity increased during each measurement in the squats, while it did not significantly change during the prone bridge (Figure 3).

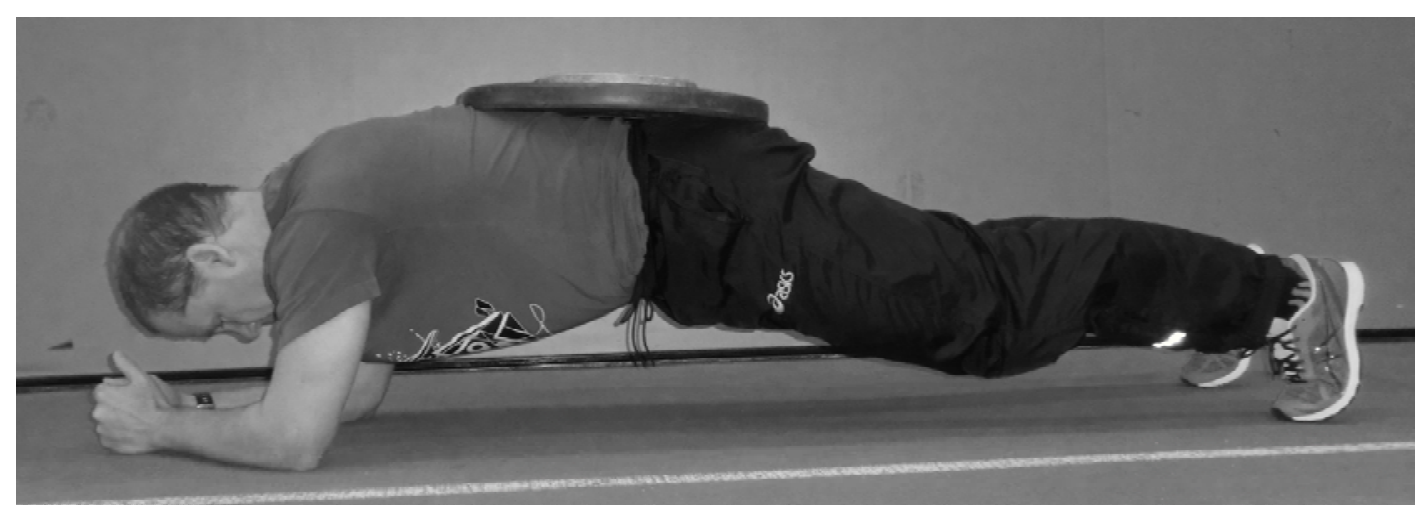

Figure 1

A prone bridge with $20 \%$ of extra body mass added to the lower back

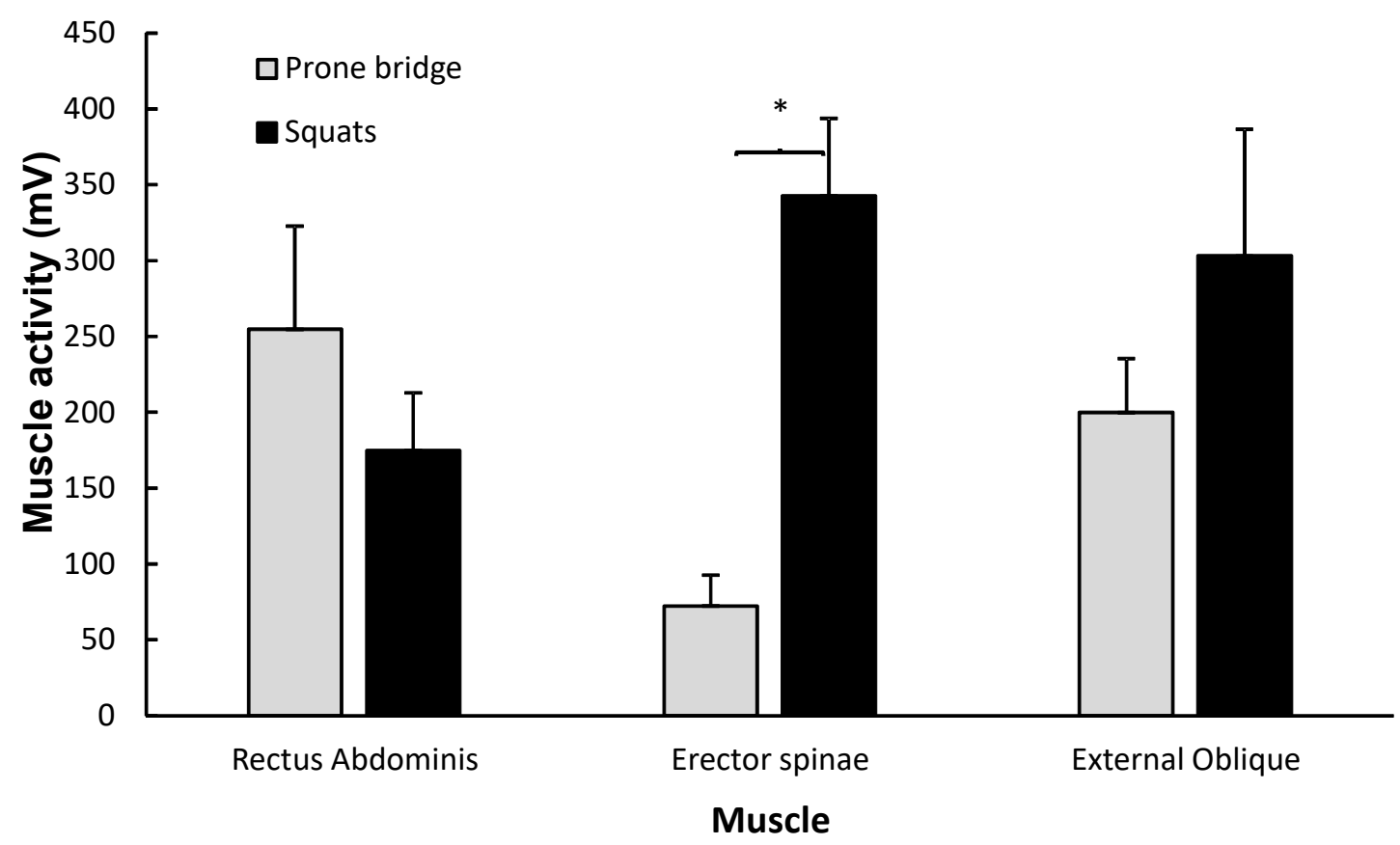

Figure 2

Mean (SEM) muscle activity of the erector spinae, rectus abdominis, and external oblique during the whole 6-RM squats and the prone bridge

* indicates a significant difference compared with the other exercises at a $p<0.05$ level 


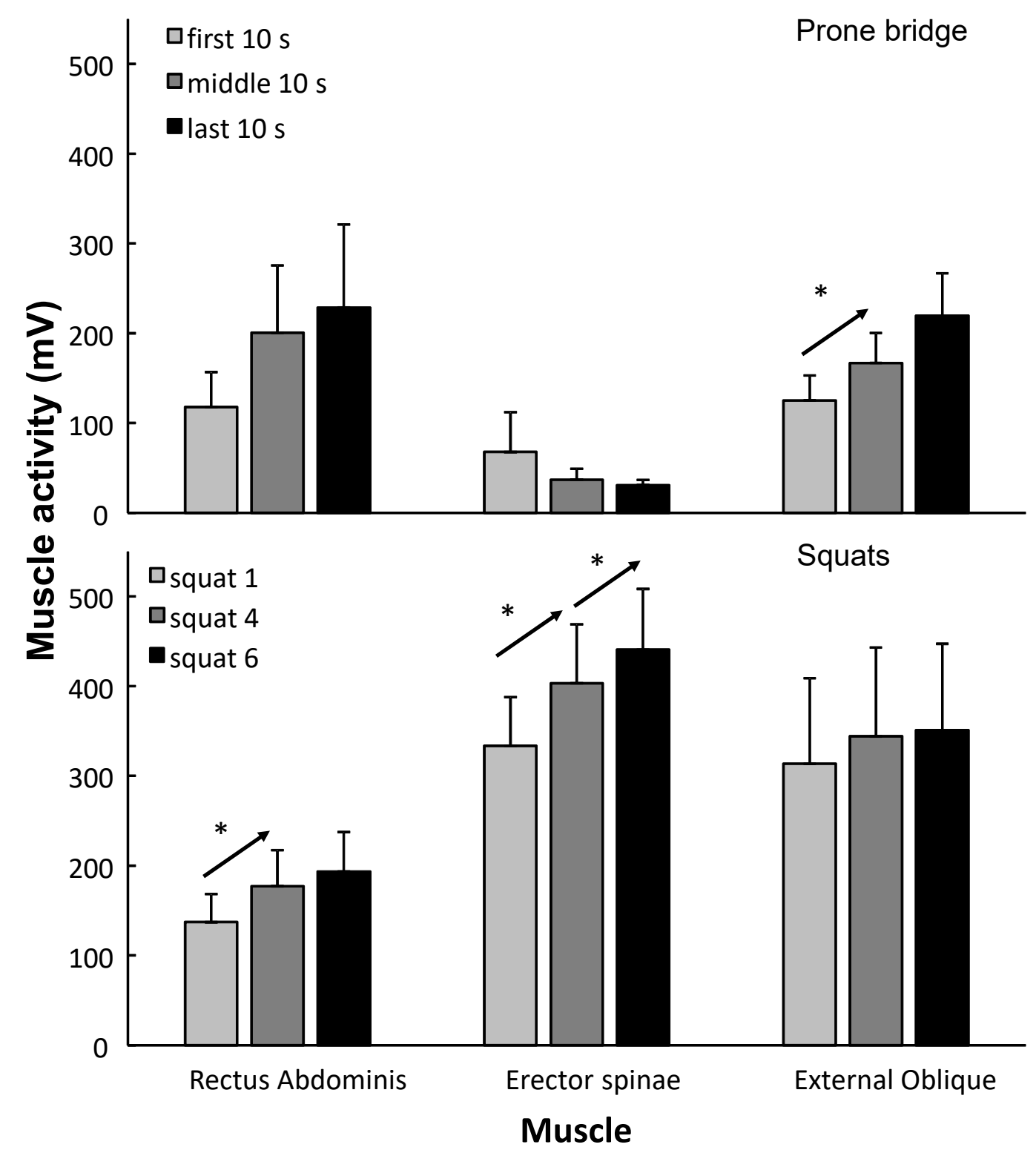

Figure 3

Mean (SEM) muscle activity of the erector spinae, rectus abdominis, and external oblique at the start, middle, and end of the 6-RM squats and the prone bridge

* indicates a significant increase from this event to the next one at a $p<0.05$ level. 


\section{Discussion}

The purpose of this study was to compare superficial core muscle activity between the prone bridge and back squats in experienced resistancetrained subjects. The main findings showed nonsignificant differences between the exercises in the rectus abdominis and external oblique, but greater erector spinae activation in squatting. In contrast to the prone bridge, the erector spinae and rectus abdominis demonstrated increased muscle activation throughout the squatting repetitions, while the prone bride demonstrated increased external oblique muscle activation between the beginning and the middle of the set.

In line with the hypotheses, the freeweight squat caused approximately four times greater muscle activation in the erector spinae than the specific core exercise that is the prone bridge. Importantly, the muscle action in the two exercises is completely different and probably it caused the differences. In squats, the erector spinae is a prime mover and synergist to extend the pelvis working dynamically (Clark et al., 2012). However, in the prone bridge, the muscle action is isometric, working in co-contraction to control the pelvis. Greater muscle activity in the erector spinae during squats is therefore not surprising, and it is supported by comparable studies (Hamlyn et al., 2007; Nuzzo et al., 2008). For example, Nuzzo et al. (2008) demonstrated $70-100 \%$ muscle activation of the MVC in the erector spinae (L1 and L5) in squats and a dead lift with an intensity of $50-100 \%$ of 1 -RM. In comparison, isolated core exercises only induced $20-45 \%$ muscle activity of the MVC (Nuzzo et al., 2008).

In the present study, there were similar muscle activities in the rectus abdominis and external oblique comparing squats and the prone bridge, while higher activation levels in the prone bridge were expected. In the prone bridge, the rectus abdominis and other frontal abdominal core muscles are the prime muscles generating isometric force to avoid sway in the lower back. For the external oblique, the muscle action is similar in both exercises (avoid rotation of the pelvis/spine). While in squats, the rectus abdominis works as an antagonist in a cocontraction to stabilize and maintain the pelvis to aid to create the abdominal pressure. That similar muscle activations were found indicates that in squats core strength requirements are already very high and that it is not necessary to target these muscles more by typical core exercises like a prone bridge. Muscle activity in the external oblique in the present study was supported by the results reported by Hamlyn et al. (2007) and Nuzzo et al. (2008), who demonstrated similar muscle activation in the external oblique between resistance exercises (squat and deadlifts) and core exercises (superman, side bridge, stability ball exercises).

The squat is a dynamic movement and mimics daily living movements as well as sport movements better than isolated and isometric contraction of the core muscles using the prone bridge exercise. Based on the results of the present study, one might therefore argue that squats are more functional than the prone bridge as the muscle synchronization requirements between core muscles constantly alter. In the descending and ascending phases in the squat, the lever arm of the spine changes continuously and thereby increases the stresses of the afferent and efferent nerve system to activate and synchronize contraction and co-contraction to stiffen and stabilize the trunk. However, heavy resistance in squats may not be the best approach among beginners as they may not stabilize and synchronize the contraction/co-contraction optimally. A safer approach, but perhaps not as functional, may be to start core training by using isolated and isometric contraction (i.e. different bridge exercises). However, the present study examined resistance-trained subjects able to lift heavy weights. Greater erector spine activation, but similar rectus abdominis and oblique external activation were observed between the squats and the prone bridge and may have been a result of the testing intensity in squats. This speculation is supported by Nuzzo et al. (2008), who demonstrated greater muscle activation only in the longissumus (90 and $100 \%$ of $1-\mathrm{RM}$ ) and multifidus (100\% of $1-\mathrm{RM})$ in squats compared to three different core exercises. However, similar muscle activation was reported with a squatting intensity of 50 and $70 \%$ of 1-RM compared to core exercises. In comparison, Hamlyn et al. (2007) demonstrated similar erector spinae activation comparing core exercises and squats with body weight. However, when squatting with an 
intensity of $80 \%$ of $1-\mathrm{RM}$ greater erector spinae muscle activation was found compared with core exercises. This demonstrates that a high training intensity is necessary to achieve greater muscle activity in resistance exercises than isolated core exercises of the lower back.

An interesting observation between the present study and other comparable ones was that squat depth, loads to fatigue, and contraction forms seemed to provide similar results comparing squats with core exercises. In the present study, the 6-RM full squat was examined. Previous studies tested quarter squats using submaximal loads (six repetitions of $80 \%$ of $1-\mathrm{RM}$ ) (Hamlyn et al., 2007) or $70^{\circ}$ knee angles using only one concentric repetition (50-100\% of 1-RM) (Nuzzo et al., 2008). Compared to a full squat, a quarter squat has a higher position of the hip in the lowest position, in which the load causes less stress on the lower lumbar region and greater stress on the quadriceps. Further, testing only the concentric phase (Nuzzo et al., 2008), the participants may stabilize and optimise the trunk position perfectly prior to the lift. From a biomechanical perspective, the greatest challenge and stress of the lumber region is in the turnover from a slow and controlled eccentric phase to maximal acceleration of the barbell in the beginning of the concentric phase. By excluding the eccentric phase (Nuzzo et al., 2008), the procedures do not reflect the training procedures and thereby it leads to lower ecological validity. Despite these differences, testing squats with an intensity of $80 \%$ of 1-RM demonstrated greater erector spine activation, but similar oblique external and rectus abdominis activation.

Surprisingly, during the prone bridge exercise, only the external oblique demonstrated greater muscle activation, and the elevated muscle activation was observed only between the first 10 $\mathrm{s}$ and the middle $10 \mathrm{~s}$. To the best of our knowledge, this is the first study to compare a core exercise to fatigue (full exhaustion) with a high-intensity squat. Previous studies have been limited by comparing muscle activation in squats with $30 \mathrm{~s}$ of contraction (Nuzzo et al., 2008) or a similar time when performing six repetitions (Hamlyn et al., 2007). Several previous studies testing exercises to fatigue have demonstrated increased muscle activation due to the Henneman's size principle (Alkner et al., 2000;
McBride et al., 2010; van den Tillaar and Saeterbakken, 2014). Further, we expected increased muscle activation in the prone bridge, as extra loads ( $20 \%$ of body mass) were added to the lower back to stress core muscles to a greater extent as subjects from a previous study in our lab reported greater pain in the elbow and shoulder muscles than in the core muscles when testing the prone bride to fatigue without extra loads. In the present study, rectus abdominis activation increased by approximately $30 \%$ throughout testing, but to a non-significant level. Furthermore, the prone bridge requires isometric contraction that may reduce the blood flow compared to dynamic contraction. In a previous study, a reduced blood flow demonstrated increased muscle activation (Loenneke et al., 2015), probably as a result of the size principle. It is well documented that an increasing intensity (load used or repetitions to failure) enhances the recruitment of motor units and thus also muscle activity almost linearly (Alkner et al., 2000).

In squats, the erector spinae demonstrated increased muscle activation between repetitions 1 and 4 , and 4 and 6, and the rectus abdominis demonstrated increased muscle activation between repetitions 1 and 4 , as hypothesized. Increasing erector spine activation throughout the sets showed that this muscle is an important prime mover in squats (Clark et al., 2012). In addition, the rectus abdominis may be an important stabilizer of the core in the turnover point from the eccentric phase to the concentric phase of the squat by maintaining abdominal pressure. The abdominal pressure is crucial to maintain the position of the trunk to reduce the risk of low back injuries, especially during highintensity resistance training (Lander et al., 1990). Therefore, the present study provides important practical knowledge in core training for strength and conditioning coaches. However, there are also inherent technical limitations with the surface EMG, as the electrodes can only provide an estimate of neuromuscular activity (Farina, 2006; Farina et al., 2004). The risks of crosstalk from neighboring muscles are present even if a small inter-electrode distance is used.

\section{Conclusion}

In conclusion, squatting resulted in greater erector spine activation, but similar rectus 
abdominis and oblique external activation as the prone bridge. Typically, specific core exercises are performed isometrically in the horizontal position, attempting to isolate the different core muscles, which does not mimic movements in sport or daily living activities. For daily living activities (e.g. stair climbing, walking, lifting, throwing, kicking etc), injury prevention, and performance improvement, we recommend targeting core muscles by integrated highintensity exercises as the squat instead of an isolated and isometric core exercise, especially for athletes. The present study found no advantages of performing the prone bridge compared to squatting.

\section{References}

ACSM. American College of Sports Medicine position stand. Progression models in resistance training for healthy adults. Med Sci Sports Exerc, 2009; 41: 687-708

Alkner BA, Tesch PA, Berg HE. Quadriceps EMG/force relationship in knee extension and leg press. Med Sci Sports Exerc, 2000; 32: 459-463

Arnason A, Sigurdsson SB, Gudmundsson A, Holme I, Engebrestsen L, Bahr R. Risk factors for injuries in football. Am J Sports Med, 2004; 32: 6-16

Behm DG, Muehlbauer T, Kibele A, Granacher U. Effects of Strength Training Using Unstable Surfaces on Strength, Power and Balance Performance Across the Lifespan: A Systematic Review and Metaanalysis. Sports Med, 2015; 45: 1645-1669

Borghuis J, Hof AL, Lemmink KAPM. The Importance of Sensory-Motor Control in Providing Core Stability. Sports Med, 2008; 38: 893-916

Brennecke A, Leone R, Cadarci M, Mochizuki L, Simao R, Amadio A, Serrao JC. Neuromuscular activity during bench pres exercise performed with and without the preexhaustion method. J Strength Cond Res, 2009; 23: 1933-1940

Clark DR, Lambert MI, Hunter AM. Muscle activation in the loaded free barbell squat: a brief review. J Strength Cond Res, 2012; 26: 1169-1178

Clark KM, Holt LE, Sinyard J. Electromyographic comparison of the upper and lower rectus abdominis during abdominal exercises. J Strength Cond Res, 2003; 17: 475-483

Cohen J. Statistical Power Analysis for the Behavioral Sciences. Hillsdale, NJ, England: Lawrence Erlbaum Associates; 1988

Comfort P, Pearson SJ, Mather D. An electromyographical comparison of trunk muscle activity during isometric trunk and dynamic strengthening exercises. J Strength Cond Res, 2011; 25: 149-154

Czaprowski D, Afeltowicz A, Gębick A, Pawłowska P, Kędra A, Barrios C, Hadała M. Abdominal muscle EMG-activity during bridge exercises on stable and unstable surfaces. Phys Ther Sports, 2014; 15: 162168

Ekstrom RA, Donatelli RA, Carp KC. Electromyographic analysis of core trunk, hip, and thigh muscles during 9 rehabilitation exercises. J Orthop Sports Phys Ther, 2007; 37: 754-762

Escamilla RF, Babb E, Jew P, Kelleher P, Burnham T, Busch J, D'Anna K, Mowbray R, Imamura R. Electromyographic Analysis of Traditional and Nontraditional Abdominal Exercises: Implications for Rehabilitation and Training. Phys Ther, 2006; 86: 656-671

Farina D. Interpretation of the surface electromyogram in dynamic contractions. Exerc Sport Sci Rev, 2006; 34: 121-127

Farina D, Merletti R, Indino B, Graven-Nielsen T. Surface EMG crosstalk evaluated from experimental recordings and simulated signals. Reflections on crosstalk interpretation, quantification and reduction. Methods Inf Med, 2004; 43: 30-35 
Gentil P, Oliveira E, Rocha Junior VA, do Carmo J, Bottaro M. Effects of exercise order on upper-body muscle activation and exercise performance. J Strength Cond Res, 2007; 21: 1082-1086

Gerdle B, Karlsson S, Crenshaw AG, Elert J, Friden J. The influences of muscle fibre proportions and areas upon EMG during maximal dynamic knee extensions. Eur J Appl Physiol Occup Physiol, 2000; 81: 2-10

Gottschall JS, Mills J, Hastings B. Integration core exercises elicit greater muscle activation than isolation exercises. J Strength Cond Res, 2013; 27: 590-596

Hamlyn N, Behm DG, Young WB. Trunk muscle activation during dynamic weight-training exercises and isometrci instability activities. J Strength Cond Res, 2007; 21: 1108-1112

Hermens HJ, Freriks B, Disselhorst-Klug C, Rau G. Development of recommendations for SEMG sensors and sensor placement procedures. J Electromyogr Kinesiol, 2000; 10: 361-374

Kibler WB, Press J, Sciascia A. The role of core stability in athletic function. Sports Med, 2006; 36: 189-198

Lander JE, Simonton RL, Giacobbe JK. The effectiveness of weight-belts during the squat exercise. Med Sci Sports Exerc, 1990; 22: 117-126

Lederman E. The myth of core stability. J Body Mov Ther, 2010; 14: 84-98

Lindström B, Karlsson S, Lexell J. Isokinetic torque and surface electromyography during fatiguing muscle contractions in young and older men and women. Isokin Exerc Sci, 2006; 14: 225-234

Loenneke JP, Kim D, Fahs CA, Thiebaud RS, Abe T, Larson RD, Bemben DA, Bemben MG. Effects of exercise with and without different degrees of blood flow restriction on torque and muscle activation. Muscle Nerve, 2015; 51: 713-721

McBride JM, Larkin TR, Dayne AM, Haines TL, Kirby TJ. Effect of absolute and relative loading on muscle activity during stable and unstable squatting. Int J Sports Physiol Perf, 2010; 5: 177-183

McGill SM. Low back stability: from formal description to issues for performance and rehabilitation. Exerc Sport Sci Rev, 2001; 29: 26-31

Monfort-Panego M, Vera-Garcia FJ, Sanchez-Zuriaga D, Sarti-Martinez MA. Electromyographic studies in abdominal exercises: a literature synthesis. J Manipulative Physiol Ther, 2009; 32: 232-244

Nuzzo JL, McCaulley GO, Cormie P, Cavill MJ, McBride JM. Trunk muscle activity during stability ball and free weight exercises. J Strength Cond Res, 2008; 22: 95-102

Oliver GD, Stone AJ, Plummer H. Electromyographic examination of selected muscle activation during isometric core exercises. Clin J Sport Med, 2010; 20: 452-457

Rahimi R. Effect of different rest intervals on the exercise volume completed during squat bouts. J Sports Sci Med, 2005; 4: 361-366

Roth R, Donath L, Zahner L, Faude O. Muscle Activation and Performance During Trunk Strength Testing in High-Level Female and Male Football Players. J Appl Biomech, 2016; 32: 241-247

Saeterbakken AH, Andersen V, van den Tillaar R. Comparison of Kinematics and Muscle Activation in FreeWeight Back Squat With and Without Elastic Bands. J Strength Cond Res, 2016; 30: 945-952

Saeterbakken AH, van den Tillaar R, Seiler S. Effect of core stability training on throwing velocity in female handball players. J Strength Cond Res, 2011; 25: 712-718

Schoenfeld BJ, Contreras B, Tiryaki-Sonmez G, Willardson JM, Fontana F. An electromyographic comparison of a modified version of the plank with a long lever and posterior tilt versus the traditional plank exercise. Sports Biomech, 2014; 13: 296-306

Snarr RL, Esco MR. Electromyographic comparison of traditional and suspension push-ups. J Hum Kinet, 2013; 39: 75-83

Snarr RL, Esco MR. Electromyographical comparison of plank variations performed with and without instability devices. J Strength Cond Res, 2014; 28: 3298-3305 
Sumiaki M, Takumi T, Yohei T, Hiroaki K. Trunk Muscle Activities during Abdominal Bracing: Comparison among Muscles and Exercises. J Sports Sci Med, 2013; 12: 467-474

Tong TK, Shing W, Jinlei N. Sport-specific endurance plank test for evaluation of global core muscle function. Phys Ther Sports, 2014; 15: 58-63

van den Tillaar R. Kinematics and muscle activation around the sticking region in free weight barbell back squat. Kinesiol Slov, 2015; 21: 15-25

van den Tillaar R, Andersen V, Saeterbakken AH. The existence of a sticking region in free weight squats. J Hum Kinet, 2014; 42: 63-71

van den Tillaar R, Saeterbakken A. Effect of Fatigue Upon Performance and Electromyographic Activity in 6RM Bench Press. J Hum Kinet, 2014; 40: 57-65

\section{Corresponding author:}

\section{Roland van den Tillaar PhD.}

Department of Sports Science and Physical Education

Nord University

Odins veg 23

7603 Levanger

Norway

Phone: +47-5767 1883

Fax: 0047-7411 2001

E-mail: roland.v.tillaar@nord.no 